\section{Prevalência da fluorose dentária em escolares de 12 e 15 anos de idade em Salvador, Bahia, Brasil, 2001}

\author{
Prevalence of dental fluorosis in 12 \\ and 15 years old schoolchildren in Salvador, \\ Bahia, Brazil, 2001
}

Maria Cristina Teixeira Cangussu 1 Roberto Augusto Castellanos Fernandez 2 Cristiane Castro Rivas 3

Cláudio Ferreira Jr. 3 Luciano Cincurá Silva Santos 3

\footnotetext{
1 Departamento de Odontologia Social, Faculdade de Odontologia, Universidade Federal da Bahia,Salvador, Brasil. 2 Departamento de Práticas de Saúde Pública, Faculdade de Saúde Pública, Universidade de São Paulo, São Paulo, Brasil.

3 Faculdade de Odontologia, Universidade Federal da Bahia,Salvador, Brasil.

Correspondência Maria Cristina Teixeira Cangussu

Departamento de Odontologia Social, Faculdade de Odontologia, Universidade Federal da Bahia. Rua Araújo Pinho 62, Salvador, BA 41150-100, Brasil. cristinacangussu@lognet. com.br
}

\begin{abstract}
This study aimed to determine the prevalence of dental fluorosis in a sample of 3,313 schoolchildren (ages 12 and 15) in a health district in Salvador, Bahia, Brazil. A cross-sectional study was developed, and data were collected by seven examiners (kappa $=0.69)$ according to WHO criteria (the Dean index). Additional information such as participation in programs, access to a dentist, place of residence from 0-5 years of age, sex, and ethnicity were obtained through a questionnaire. A descriptive analysis was performed for the municipality as a whole and for each district. According to the results, $31.4 \%$ of the 12 year-olds and $27.6 \%$ of the 15-year-olds presented fluorosis, predominantly of the "very mild" type. Differences were found between the districts ( $p$ <.05). The districts of Pau da Lima and Brotas had the highest percentages of healthy individuals at 12 years of age (81.8\% and $81.6 \%$ ). At 15 years, Subúrbio Ferroviário, Pau da Lima, and Cajazeiras were the districts with the lowest prevalence rates $(13.0 \%, 14.6 \%$, and $13.5 \%)$. The district of Liberdade had the highest prevalence (72.4\% and 57.3\%) at 12 and 15 years, respectively, attributed to differences in calibration. Differences were found between the health districts, thus emphasizing the need for health surveillance.
\end{abstract}

Oral Health; Dental Fluorosis; Prevalence; Cross-Sectional Studies

\section{Introdução}

A fluorose dentária origina-se da exposição do germe dentário, durante o seu processo de formação em altas concentrações do íon flúor. Como conseqüência, têm-se defeitos de mineralização do esmalte dentário, com alterações de coloração e forma, com severidade diretamente associada à quantidade ingerida e tempo de consumo 1 .

Discute-se, atualmente, a possibilidade de um maior risco do acometimento da população pela difusão maciça do uso dos fluoretos 2 , especialmente os dentifrícios fluoretados, que tiveram o seu uso regulamentado no país somente na década de 90. Entretanto, Souza et al. 3 descrevem a dificuldade no país de garantir o acesso ao uso continuado do flúor, seja pelo uso dos dentifrícios, seja por outros veículos de uso sistêmico ou tópico, devido às grandes diferenças sociais e econômicas que têm como conseqüência acesso diferenciado aos bens públicos, serviços de saúde, além de aquisição de produtos fluorados de uso domiciliar.

Em um reduzido número de estudos envolvendo a população brasileira, observou-se um aumento da ocorrência da fluorose dentária. Pereira et al. 4 descrevem em Piracicaba, município com água fluoretada, e Iracemápolis, sem água fluoretada, um incremento de 52\% e $41 \%$ em relação a levantamentos anteriores. Já Maltz et al. 5, em Porto Alegre, verificaram o aumento 
de $24 \%$ da fluorose dentária no período de 1987 a 1997 em crianças de 8-9 anos de idade, embora os autores coloquem que o aumento da prevalência tenha sido nas formas mais leves, não implicando risco à saúde.

Dentre os estudo de prevalência de fluorose dentária publicados no Brasil, utilizando o índice de Dean, tem-se o de Narvai et al. 6, que examinaram 2.491 crianças de escolas públicas e privadas, aos 12 anos de idade do Município de São Paulo, e relataram uma prevalência de $21,8 \%$, com $3,7 \%$ correspondendo ao grau moderado. No interior do Estado de São Paulo, Gaspar et al. 7 analisaram 211 crianças em Piracicaba e 200 em Iracemápolis, nas idades de 10 a 14 anos, encontrando uma prevalência de $20,9 \%$ e $4,5 \%$ de fluorose, respectivamente. Buendia \& Zaina 8, em Presidente Prudente, estudaram 1.053 crianças de 8 a 13 anos, tendo como resultado uma prevalência de $17,5 \%$. Já Marcelino et al. 9, em Araçatuba, relatam 24,0\% de fluorose dentária em 317 crianças de 12 a 14 anos de idade.

Em 1998, a Secretaria de Estado da Saúde de São Paulo, em parceria com universidades, desenvolveu o Levantamento Epidemiológico em Saúde Bucal, oportunidade em que a fluorose dentária também foi avaliada em escolares de 5, 12 e 18 anos de idade. Para todo o Estado foi descrita uma prevalência de $11,2 \%$ desta alteração aos 12 anos e 7,9\% aos 18 anos. Os percentuais tiveram variação nos municípios com e sem água de abastecimento público fluoretada, correspondendo a $15,2 \%$ e $5,0 \%$, respectivamente, em crianças de 12 anos de idade. Em praticamente todas as Diretorias Regionais de Saúde (DIR), a prevalência de fluorose não excedeu $20,0 \%$ nas idades estudadas, salvo a da DIR II (Santo André), que aos 12 anos apresentou um valor de $82,8 \%$, discrepante com o resultado descrito nesta mesma região aos 18 anos e também no restante do Estado 10.

Silva \& Paiva 11, em Belo Horizonte, fizeram um levantamento em 518 escolares entre 7 e 14 anos de idade e verificaram uma prevalência de 25,5\% desta alteração. Já Mendonça et al. 12, neste mesmo Município, relataram uma prevalência de $34,4 \%$ ao examinarem 797 escolares de 7 a 10 anos.

Alcântara 13, também utilizando o Índice de Dean para mensurar a fluorose dentária em 360 crianças de 7 a 14 anos, de escolas públicas de Curitiba, relata uma prevalência de $25,6 \%$, com predominância do grau muito leve, representando $13.9 \%$.

Em Salvador, Reis et al. 14 realizaram um levantamento da prevalência de fluorose dentária (Índice de Dean) em quatro escolas, duas públicas e duas privadas, com crianças de sete anos de idade, identificando uma prevalência de $20,1 \%$, com predominância de casos de fluorose moderada.

A fluoretação das águas de abastecimento público em Salvador foi introduzida em 1975 e interrompida em 1989, ainda que não existam, neste período, registros de controle do nível ótimo de flúor, a não ser o fornecido pela empresa pública de abastecimento de água, estabelecido em $0.7 \mathrm{ppm}$. O retorno ao processo de fluoretação se deu em novembro de 1996, com dosagens entre 0.7-0.8ppm e, concomitantemente, a estruturação de um sistema de vigilância sanitária, trabalho realizado pela Universidade, pelo Conselho Regional de Odontologia e pela Vigilância Sanitária Municipal 15. O percentual da população com acesso à água tratada era de $85,4 \%$ no início da década de 80 e de $93,87 \%$ em 199116

Neste sentido, o objetivo deste trabalho foi descrever a prevalência de fluorose dentária na população escolar de 12 e 15 anos de idade do Município de Salvador, de acordo com a distribuição geográfica por distrito sanitário, de modo a identificar as áreas prioritárias de risco e intervenção, contribuindo assim para o planejamento local em saúde bucal e implantação de novas ações de vigilância à saúde.

\section{Metodologia}

Desenvolveu-se um estudo de prevalência, utilizando como população os estudantes de escolas públicas e privadas do Município de Salvador. Em 2000, a cidade possuía uma população de 2.440.886 habitantes, distribuídos em 12 Distritos Sanitários, sendo eles: Barra; Rio Vermelho; Centro Histórico; Itapagipe; Brotas; Boca do Rio; Itapoã; Cajazeiras; Cabula-Beiru; Liberdade; Pau da Lima; Subúrbio Ferroviário e São Caetano; Valéria 17. Para este estudo foram recuperadas as mensurações dos teores de flúor na água nos 12 distritos sanitários, e estes variaram de $0.61 \mathrm{ppmF}$ a $0.73 \mathrm{ppmF}$.

Utilizou-se para este trabalho uma amostra probabilística em múltiplo estágio, com unidades amostrais primárias de escolas públicas e particulares da cidade 18 . Foram sorteados 72 estabelecimentos de ensino do total de 778, préestratificados por Distrito Sanitário, obedecendo à proporcionalidade entre o setor público e privado, com a ponderação da amostra de acordo com a densidade populacional de cada distrito, processo descrito em artigo anterior 19.

A coleta de dados foi realizada em ambiente escolar, após terem sido obtidos os termos 
de consentimento dos pais, obtendo-se os dados gerais por intermédio de um questionário aplicado pelo anotador e os dados de fluorose dentária pelo exame clínico oral:

- Dados gerais para caracterização da população de estudo: nome, data de nascimento, endereço da residência, nome da escola, tipo de financiamento da escola (pública, privada), distrito sanitário em que a escola estava localizada, local de moradia nos primeiros cinco anos de vida, acesso à atenção à saúde bucal, participação em outros programas com uso de flúor tópico nos últimos dois anos (sim/não), acesso à assistência odontológica no último ano (sim/não) e tipo de financiamento da mesma (se público, particular ou convênio).

- Exame clínico: foram inspecionados todos os dentes e aplicados os critérios estabelecidos pela Organização Mundial da Saúde para o diagnóstico da fluorose dentária, utilizando o Índice de Dean, isto é, com iluminação natural, sem profilaxia prévia e uso de espelho clínico e sonda periodontal da OMS com a criança em decúbito dorsal 20 .

Os dados foram coletados por sete examinadores previamente calibrados, tendo sido mensurado no momento do treinamento, os erros inter e intra-examinador. A concordância entre os examinadores foi de $89,72 \%$, com um kappa $=0,69$, e a menor concordância intra examinador de $92,01 \%$.

O desenvolvimento deste estudo seguiu os requisitos éticos da Resolução 196/96 do Conselho Nacional de Saúde, Ministério da Saúde do Brasil 21, e foi aprovado pelo Comitê de Ética da Faculdade de Saúde Pública da Universidade de São Paulo.

Os dados foram digitados e armazenados num banco de dados criado no programa Epi Info, versão 6.04. Os cálculos dos erros de digitação foram feitos com base na dupla entrada de dados e percentual de concordância das mesmas, bem como a revisão do banco após a identificação de erros na análise descritiva de cada variável. A análise foi realizada no Epi Info e no pacote estatístico STATA, versão 6.0.

Deu-se seguimento à análise descritiva da prevalência e severidade da fluorose dental para o Município de Salvador e por distrito sanitário de localização da escola; e à identificação de distritos homogêneos na distribuição desta alteração valendo-se do teste do qui-quadrado, com um nível de significância de 5\% 22 .

\section{Resultados}

Foram inspecionados um total de 3.318 adolescentes, e após a revisão e digitação das fichas, foram excluídas cinco por falta de preenchimento do campo "idade". Portanto, foram 3.313 as fichas utilizadas efetivamente para análise, sendo 1.750 aos 12 anos e 1.563 aos 15 anos. O erro de digitação foi de $0,2 \%$ dos campos, e, após a identificação dos mesmos, retornou-se às fichas originais, limpando o banco de dados.

As perdas amostrais tanto aos 12 quanto aos 15 anos de idade foram pequenas, representando $0,9 \%$ da amostra sorteada, não tendo sido necessária a reposição de alunos em função das recusas de participação ou ausência no momento do exame. Quando somados os dados excluídos e as perdas de participação, o percentual obtido totalizou 1,3\%.

O número de estudantes do sexo feminino examinado foi maior do que aqueles do sexo masculino. Houve a predominância na amostra da etnia parda, $71,2 \%$ aos 12 anos e $73,2 \%$ aos 15 anos, seguida da negra e branca, respectivamente. $\mathrm{O}$ acesso ao dentista no último ano aproximou-se dos 50\% da população de estudo, com uma distribuição semelhante em relação ao tipo de financiamento - público, particular e convênio. A participação em programas com o uso do flúor em escolas (escovação supervisionada com uso do flúor) nos últimos 2 anos foi maior aos 12 anos $(39,7 \%)$ do que aos 15 anos de idade $(27,6 \%)$. A maior proporção da amostra constituiu-se de estudantes nascidos e residentes na infância no próprio Município de Salvador, $84,7 \%$ aos 12 anos, caracterizando neste grupo a não-ingestão de flúor sistêmico decorrente da fluoretação das águas de abastecimento público até 1996, enquanto aos 15 anos, estes alunos estiveram apenas parcialmente (dos 0 aos 3 anos de idade) submetidos a este método preventivo (Tabela 1 ).

A prevalência de fluorose encontrada no Município de Salvador foi de 31,4\% aos 12 anos de idade e 27,6\% aos 15 anos de idade, com uma predominância dos casos no grau muito leve, conforme se observa na Tabela 2.

Foram encontradas diferenças estatisticamente significativas (teste do qui-quadrado; nível de significância de 5\%) na análise descritiva da distribuição da fluorose dentária segundo distrito sanitário, com menores prevalências, aos 12 anos de idade, para as regiões: Barra-Rio Vermelho, Brotas, Itapoã, Cabula-Beiru e Pau da Lima, com percentuais abaixo dos 30\%. Já aos 15 anos de idade, os distritos de Brotas, Boca do Rio, Cajazeiras, Cabula-Beiru, 
Tabela 1

Caracterização da população de estudo aos 12 e 15 anos segundo tipo de escola, sexo, etnia, visita ao dentista no último ano, tipo de financiamento da mesma, participação em programas com uso do flúor e local de moradia nos primeiros 5 anos de vida. Salvador, Bahia, Brasil, 2001.

\begin{tabular}{|c|c|c|c|c|}
\hline \multirow[t]{2}{*}{ Variáveis } & \multicolumn{2}{|c|}{$\begin{array}{c}12 \text { anos } \\
(n=1.750)\end{array}$} & \multicolumn{2}{|c|}{$\begin{array}{c}15 \text { anos } \\
(n=1.563)\end{array}$} \\
\hline & $\mathrm{n}$ & $\%$ & $\mathrm{n}$ & $\%$ \\
\hline \multicolumn{5}{|l|}{ Tipo de Escola } \\
\hline Pública & 1.315 & 75,1 & 1.139 & 72,9 \\
\hline Particular & 435 & 24,9 & 424 & 27,1 \\
\hline \multicolumn{5}{|l|}{ Sexo } \\
\hline Masculino & 797 & 45,5 & 661 & 42,3 \\
\hline Feminino & 953 & 54,5 & 902 & 57,7 \\
\hline \multicolumn{5}{|l|}{ Etnia } \\
\hline Branco & 88 & 5,0 & 73 & 4,7 \\
\hline Negro & 414 & 23,7 & 345 & 22,1 \\
\hline Pardo & 1.247 & 71,2 & 1.145 & 73,2 \\
\hline Amarelo & 1 & 0,1 & - & - \\
\hline \multicolumn{5}{|c|}{ Visita ao dentista no último ano } \\
\hline Sim & 902 & 51,5 & 741 & 47,4 \\
\hline Não & 784 & 44,8 & 780 & 49,9 \\
\hline Não sabe & 64 & 3,7 & 42 & 2,7 \\
\hline \multicolumn{5}{|l|}{ Financiamento } \\
\hline Público & 303 & 17,3 & 206 & 13,2 \\
\hline Particular & 279 & 15,9 & 268 & 17,1 \\
\hline Convênio & 250 & 14,3 & 242 & 15,5 \\
\hline Não sabe & 86 & 4,9 & 33 & 2,1 \\
\hline Não se aplica & 832 & 47,6 & 814 & 52,1 \\
\hline \multicolumn{5}{|c|}{$\begin{array}{l}\text { Participação programas } \\
\text { coletivos últimos } 2 \text { anos }\end{array}$} \\
\hline Sim & 695 & 39,7 & 432 & 27,6 \\
\hline Não & 985 & 56,3 & 1.057 & 67,7 \\
\hline Não sabe & 70 & 4,0 & 74 & 4,7 \\
\hline \multicolumn{5}{|c|}{$\begin{array}{l}\text { Local de moradia dos } 0 \\
\text { aos } 5 \text { anos de idade }\end{array}$} \\
\hline Salvador & 1.481 & 84,7 & 1.278 & 81,8 \\
\hline Outros & 269 & 15,3 & 285 & 18,2 \\
\hline
\end{tabular}

Pau da Lima, Subúrbio Ferroviário e São Caetano-Valéria apresentaram prevalências abaixo dos $30 \%$ (Tabelas 3 e 4).

Uma alta prevalência desta alteração foi descrita no distrito da Liberdade, com um percentual de $72,4 \%$ ao 12 anos e, aos 15 anos de idade, esta tendência repete-se com um percentual de $57,3 \%$ de indivíduos com algum grau de fluorose dentária. O Centro Histórico e Itapagipe tiveram como resultado percentuais entre $30,0 \%$ e $40,0 \%$ tanto aos 12 quanto aos 15 anos de idade. Esta mesma faixa de prevalência repetiu-se nas regiões do Barra-Rio Vermelho e Itapoã aos 15 anos de idade. Os distritos com menores proporções desta alteração foram aos 12 anos - Pau da Lima (18,2\%), Brotas $(18,4 \%)$ e Cabula-Beiru (19,4\%), e aos 15 anos Subúrbio Ferroviário (13,0\%), Cajazeiras (13,5\%) e Pau da Lima $(14,6 \%)$.

\section{Discussão}

As prevalências de fluorose dentária encontradas aos $12(31,4 \%)$ e aos 15 anos de idade $(27,6 \%)$ estão próximas do percentual de $30,0 \%$, valor descrito na maior parte dos estudos feitos no Brasil, em regiões com água de abastecimento público fluoretada em níveis ótimos, com o predomínio também da categoria de severidade "muito leve" 6,7,8,11.

Significa que, apesar da necessidade de vigilância à saúde em relação a esta alteração em função do risco potencial do incremento da mesma decorrente da difusão maciça de fluoretos, como descrevem Clarck et al. 2, e do retorno da fluoretação das águas no município no final de 1996, tal fluoretação ainda não pode ser considerada um problema de saúde pública, como também descrevem os resultados do levantamento epidemiológico realizado no Estado de São Paulo 10, no estudo de Alcântara13 em Curitiba, e de Silva \& Paiva11 em Belo Horizonte, Minas Gerais, todos envolvendo populações de escolares.

Identificou-se, em relação ao estudo anterior desenvolvido no município por Reis et al.14, discrepâncias na prevalência da fluorose dentária, já que neste estudo foram quase inexistentes os casos de fluorose moderada. Esta diferença pode ser atribuída às especificidades das populações de estudo examinadas - o estudo destes autores investigaram apenas quatro escolas na idade de sete anos de idade, enquanto o atual estudo envolveu uma amostra da população escolar aos 12 e 15 anos de idade; aos dentes examinados - apenas os incisivos centrais e todo o arco dentário, respectivamente; a 
subjetividade inerente ao exame da fluorose dentária, visto que os examinadores não foram os mesmos em ambos os estudos; bem como as diferenças nas condições do exame.

As menores prevalências de fluorose dentária foram encontradas nos distritos de Brotas e Pau da Lima, aos 12 anos de idade, e Pau da Lima e Cajazeiras aos 15 anos de idade. Detectaram-se diferenças estatisticamente significantes (5\%) entre as áreas no teste do qui-quadrado, com maiores prevalências para o distrito da Liberdade, em ambas as idades.

Ocorrências mais altas como as identificadas em alguns distritos neste estudo têm sido atribuídas ao efeito combinado do uso e ingestão de produtos dentários de outras fontes sistêmicas de flúor, bem como as diferenças no acesso aos bens públicos (como o saneamento básico) e de consumo individual 1,12.

Por mais que esta seja uma possível explicação para as diferenças entre as regiões, vale ressaltar que as discrepâncias encontradas nos distritos da Liberdade, Pau da Lima e Brotas, aos 12 anos de idade, e Liberdade, Pau da Lima, Cajazeiras e Subúrbio Ferroviário, aos 15 anos de idade, indicam a possibilidade de diferenças na interpretação da fluorose dentária entre os examinadores. Os exames na Liberdade foram realizados por um único examinador, enquanto outro examinador responsabilizou-se pelos distritos de Pau da Lima e Cajazeiras. Isto é, mesmo que tenha sido feita a calibração da equipe e tenham sido obtidos coeficientes de concordância satisfatórios 20 , a subjetividade inerente ao estudo da fluorose dentária pode ter interferido na análise por áreas.

Além disso, como o cálculo amostral foi realizado, no estudo original, pela utilização dos indicadores de cárie como referência, na análise da fluorose dentária, esta mostrou-se insuficiente para produzir estimativas mais precisas em cada área geográfica, podendo ter sido afetada também por escolha de grupos específicos de maior risco para esta alteração.

Destaca-se a relevância, com base neste trabalho, do desenvolvimento de estudos epidemiológicos continuados no Município de Salvador, como forma a avaliar o impacto da re-

Tabela 2

Distribuição da fluorose dentária (Índice de Dean) aos 12 e 15 anos de idade. Salvador, Bahia, Brasil, 2001.

\begin{tabular}{lrrrrrr}
\hline \multirow{2}{*}{ Grau } & \multicolumn{2}{c}{12 anos } & \multicolumn{2}{c}{15 anos } & \multicolumn{2}{c}{ Total } \\
& \multicolumn{1}{c}{$\mathrm{n}$} & $\%$ & $\mathrm{n}$ & $\%$ & $\mathrm{n}$ & $\%$ \\
\hline Sem Fluorose & 1.200 & 68,6 & 1.132 & 72,4 & 2.332 & 70,4 \\
Normal & 996 & 56,9 & 935 & 59,8 & 1931 & 58,3 \\
Questionável & 204 & 11,7 & 197 & 12,6 & 401 & 12,1 \\
Com fluorose & 550 & 31,4 & 431 & 27,6 & 981 & 29,6 \\
Muito leve & 478 & 27,3 & 365 & 23,4 & 843 & 25,4 \\
Leve & 69 & 3,9 & 63 & 4,0 & 132 & 4,0 \\
Moderado & 3 & 0,2 & 3 & 0,2 & 6 & 0,2 \\
Severo & - & - & - & - & - & - \\
Total & 1.750 & 100,0 & 1.563 & 100,0 & 3.313 & 100,0 \\
\hline
\end{tabular}

Tabela 3

Percentual de casos de fluorose dentária (Índice de Dean) em escolares de 12 anos de idade segundo distrito sanitário. Salvador, Bahia, Brasil, 2001.

\begin{tabular}{|c|c|c|c|c|c|c|c|c|c|c|c|c|}
\hline \multirow{2}{*}{$\begin{array}{l}\text { Graus de } \\
\text { fluorose }\end{array}$} & \multicolumn{12}{|c|}{ Distrito Sanitário } \\
\hline & $\begin{array}{c}\text { BRV } \\
(n=202)\end{array}$ & $\begin{array}{c}\mathrm{CH} \\
(\mathrm{n}=134)\end{array}$ & $\begin{array}{c}\text { ITG } \\
(n=150)\end{array}$ & $\begin{array}{c}\text { BRT } \\
(n=141)\end{array}$ & $\begin{array}{c}\text { BR } \\
(n=146)\end{array}$ & $\begin{array}{c}\text { ITA } \\
(n=127)\end{array}$ & $\begin{array}{c}\text { CAJ } \\
(n=140)\end{array}$ & $\begin{array}{c}C B \\
(n=144)\end{array}$ & $\begin{array}{c}\text { LIB } \\
(n=112)\end{array}$ & $\begin{array}{c}P L \\
(n=137)\end{array}$ & $\begin{array}{c}S F \\
(n=164)\end{array}$ & $\begin{array}{c}\text { SCV } \\
(n=153)\end{array}$ \\
\hline Sem fluorose & 73,2 & 59,7 & 68,0 & 81,6 & 60,3 & 70,1 & 71,5 & 80,6 & 27,6 & 81,8 & 68,9 & 69,3 \\
\hline Normal & 59,3 & 38,1 & 56,7 & 77,3 & 45,9 & 47,3 & 68,6 & 61,8 & 8,0 & 81,0 & 62,2 & 63,4 \\
\hline Questionável & 13,9 & 21,6 & 11,3 & 4,3 & 14,4 & 22,8 & 2,9 & 18,8 & 19,6 & 0,8 & 6,7 & 5,9 \\
\hline Com fluorose & 26,8 & 40,3 & 32,0 & 18,4 & 39,7 & 29,9 & 28,5 & 19,4 & 72,4 & 18,2 & 31,1 & 30,7 \\
\hline Muito leve & 24,3 & 35,1 & 27,3 & 17,0 & 32,2 & 25,2 & 27,1 & 12,5 & 55,4 & 18,2 & 29,9 & 30,1 \\
\hline Leve & 2,5 & 5,2 & 4,0 & 1,4 & 6,8 & 3,9 & 1,4 & 6,9 & 17,0 & - & 1,2 & 0,6 \\
\hline Moderada & - & - & 0,7 & - & 0,7 & 0,8 & - & - & - & - & - & - \\
\hline Severa & - & - & - & - & - & - & - & - & - & - & - & - \\
\hline Total & 100,0 & 100,0 & 100,0 & 100,0 & 100,0 & 100,0 & 100,0 & 100,0 & 100,0 & 100,0 & 100,0 & 100,0 \\
\hline
\end{tabular}

* Diferenças estatisticamente significativas no nível de $5 \%$ no teste do qui-quadrado.

$\mathrm{BRV}=$ Barra-Rio Vermelho; $\mathrm{CH}=$ Centro Histórico; ITG = Itapagipe; BRT = Brotas; BR = Boca do Rio;

$I T A=$ Itapoã; $C A J=$ Cajazeiras; $C B=$ Cabula-Beiru; LIB = Liberdade; PL = Pau da Lima; SF = Subúrbio Ferroviário;

$\mathrm{SCV}=$ São Caetano-Valéria. 
Tabela 4

Percentual de casos de fluorose dentária (Índice de Dean) em escolares de 15 anos de idade segundo distrito sanitário. Salvador, Bahia, Brasil, 2001.

\begin{tabular}{|c|c|c|c|c|c|c|c|c|c|c|c|c|}
\hline \multirow{2}{*}{$\begin{array}{l}\text { Graus de } \\
\text { fluorose }\end{array}$} & \multicolumn{12}{|c|}{ Distrito Sanitário } \\
\hline & $\begin{array}{c}\text { BRV } \\
(n=174)\end{array}$ & $\begin{array}{c}\mathrm{CH} \\
(n=137)\end{array}$ & $\begin{array}{c}\text { ITG } \\
(n=109)\end{array}$ & $\begin{array}{c}\text { BRT } \\
(n=140)\end{array}$ & $\begin{array}{c}\text { BR } \\
(n=111)\end{array}$ & $\begin{array}{c}\text { ITA } \\
(n=139)\end{array}$ & $\begin{array}{c}\text { CAJ } \\
(n=148)\end{array}$ & $\begin{array}{c}\text { CB } \\
(n=140)\end{array}$ & $\begin{array}{c}\text { LIB } \\
(n=117)\end{array}$ & $\begin{array}{c}\mathrm{PL} \\
(\mathrm{n}=110)\end{array}$ & $\begin{array}{c}\text { SF } \\
(n=131)\end{array}$ & $\begin{array}{c}\text { SCV } \\
(n=107)\end{array}$ \\
\hline Sem fluorose & 68,5 & 64,3 & 66,0 & 71,5 & 77,5 & 61,8 & 86,5 & 81,4 & 42,7 & 85,4 & 87,0 & 75,7 \\
\hline Normal & 54,0 & 41,6 & 58,7 & 67,9 & 60,4 & 41,7 & 85,1 & 61,4 & 15,4 & 84,5 & 78,6 & 69,2 \\
\hline Questionável & 14,5 & 22,7 & 7,3 & 3,6 & 17,1 & 20,1 & 1,4 & 20,0 & 27,3 & 0,9 & 8,4 & 6,5 \\
\hline Com fluorose & 31,5 & 35,7 & 34,0 & 28,5 & 22,5 & 38,2 & 13,5 & 18,6 & 57,3 & 14,6 & 13,0 & 24,3 \\
\hline Muito leve & 26,4 & 29,9 & 29,4 & 23,6 & 18,9 & 32,4 & 13,5 & 10,7 & 46,1 & 14,6 & 13,0 & 23,4 \\
\hline Leve & 4,0 & 5,8 & 4,6 & 5,0 & 3,6 & 5,8 & - & 7,9 & 10,3 & - & - & 0,9 \\
\hline Moderada & 1,1 & - & - & - & - & - & - & - & 0,9 & - & - & - \\
\hline Severa & - & - & - & - & - & - & - & - & - & - & - & - \\
\hline Total & 100,0 & 100,0 & 100,0 & 100,0 & 100,0 & 100,0 & 100,0 & 100,0 & 100,0 & 100,0 & 100,0 & 100,0 \\
\hline
\end{tabular}

* Diferenças estatisticamente significativas no nível de $5 \%$ no teste do qui-quadrado.

$\mathrm{BRV}=$ Barra-Rio Vermelho; $\mathrm{CH}=$ Centro Histórico; ITG = Itapagipe; $\mathrm{BRT}=$ Brotas; $\mathrm{BR}=$ Boca do Rio;

ITA = Itapoã; CAJ = Cajazeiras; CB = Cabula-Beiru; LIB = Liberdade; PL = Pau da Lima; SF = Subúrbio Ferroviário;

$\mathrm{SCV}=$ São Caetano-Valéria.

introdução da fluoretação das águas de abastecimento público na prevalência e severidade da fluorose dentária, bem como detectar as possíveis causas das diferenças de prevalências entre os distritos, além de identificar grupos ou áreas de maior suscetibilidade à alteração, a fim de que se construa um sistema de atenção à saúde bucal voltado para a vigilância à saúde.

\section{Conclusões}

- A prevalência de fluorose dentária no $\mathrm{Mu}$ nicípio de Salvador foi de $29,6 \%$, sendo $31,4 \%$ aos 12 anos de idade e $27,6 \%$ aos 15 anos de idade;

\section{Resumo}

O objetivo foi determinar a prevalência da fluorose dentária em uma amostra de 3.313 escolares de 12 e 15 anos em distrito sanitário em Salvador, Bahia, Brasil. Desenvolveu-se um estudo transversal e os dados foram coletados por sete examinadores (kappa $=0.69)$, segundo os critérios da OMS (índice de Dean). Informações adicionais como participação em programas, acesso ao dentista, local de moradia dos 0-5 anos de vida,sexo e etnia foram obtidos mediante questionário. Procedeu-se à análise descritiva para o município e para cada distrito. Resultou que 31,4\% dos escolares aos 12 anos $e$ 27,6\% aos 15 anos apresentavam fluorose,predominando a categoria "muito leve". Foram encontradas diferenças entre os distritos ( $\left.p<0.05 X^{2}\right)$. Os
- Aos 12 anos, os distritos com menores proporções desta alteração foram Pau da Lima $(18,2 \%)$, Brotas $(18,4 \%)$ e Cabula-Beiru $(19,4 \%)$; e aos 15 anos de idade os distritos Pau da Lima $(14,6 \%)$, Cajazeiras $(13,5 \%)$ e Subúrbio Ferroviário $(13,0 \%)$ foram os de menor prevalência; - Aos 12 e 15 anos de idade, o distrito da Liberdade foi o que apresentou maior proporção de indivíduos acometidos por fluorose dentária $-72,4 \%$ e $57,3 \%$, respectivamente, resultados discrepantes em relação ao restante do $\mathrm{Mu}$ nicípio, sendo possível que os mesmos tenham sido afetados pela calibração dos examinadores ou desenho amostral;

- A fluorose dentária parece não constituir, até o momento, um problema de saúde pública no Município de Salvador, Bahia. distritos de Pau da Lima e Brotas foram os com maior percentual de indivíduos sadios aos 12 anos $-81,8 \% e$ 81,6\%; ; aos 15 anos, Subúrbio Ferroviário, Pau da Lima e Cajazeiras foram as regiões com menor prevalência-13,0\%, 14,6\% e 13,5\%. O distrito da Liberdade apresentou maior prevalência -72,4\% e 57,3\% aos 12 e 15 anos, respectivamente, atribuída às diferenças na calibração da equipe. Foram identificadas diferenças entre os distritos sanitários, reforçando a necessidade de vigilância à saúde.

Saúde Bucal; Fluorose Dentária; Prevalência; Estudos Transversais 


\section{Colaboradores}

M. C. T. Cangussu foi responsável pela coleta de dados, sistematização do conhecimento, análise e discussão dos dados. R. A. C. Fernandez participou da revisão teórica e discussão dos dados. C. Rivas, C. Ferreira Jr. e L Santos foram responsáveis pela busca bibliográfica, parte da coleta de dados, revisão do banco de dados e elaboração e discussão dos resultados.

\section{Referências}

1. Den Besten PK. Biological mechanisms of dental fluorosis relevant to the use of fluoride supplements. Community Dent Oral Epidemiol 1999; 27:41-7.

2. Clarck DC, Hann HJ, Williamson MF, Berkowitz J. Influence of exposure to various fluoride technologies on the prevalence of dental fluorosis. Community Dent Oral Epidemiol 1994; 22:461-4.

3. Souza SMD, Cury JA, Narvai PC, Gevaerd SP. Saúde bucal: prevenção para 100 porcento da população infantil. Revista da Associação Brasileira de Odontologia 1998; 6:182-6.

4. Pereira AC, Cunha MC, Meneghim MC. Prevalência de cárie dentária e fluorose em escolares de áreas fluoretadas e não fluoretadas. 15a Reunião Anual da Sociedade Brasileira de Pesquisa Odontológica; 199813 Agosto; São Paulo, Brasil. Águas de Lindóia: Pesquisa Odontológica Brasileira; 1998.

5. Maltz M, Silva BB, Schaeffer A. Prevalência de fluorose em uma cidade brasileira com água fluoretada, em 1987 e 1997. 15a Reunião Anual da Sociedade Brasileira de Pesquisa Odontológica; 1998 13 Agosto; São Paulo, Brasil. Águas de Lindóia: Pesquisa Odontológica Brasileira; 1998.

6. Narvai PC, Castellanos RA, Frazão P. Levantamento Epidemiológico de oclusopatias e fluorose dentária em crianças de 5 a 12 anos de idade, no Município de São Paulo, em 1996. Anais do II Congresso Brasileiro de Saúde Coletiva; 1997 1-7 Julho, Águas de Lindóia, Brasil. Rio de Janeiro: ABRASCO; 1997.

7. Gaspar MR. Opacidades de origem não fluorótica e fluorose dentária em áreas com baixa $(0,2 \mathrm{ppmF})$ e ótima $(0,7 \mathrm{ppmF})$ concentração de flúor na água de abastecimento. Rev Bras Odontol 1995; 52:13-8.

8. Buendia OC, Zaina J. Será o flúor vilão? Revista Paulista de Odontologia 1997; 19:36-44.

9. Marcelino G, Guimarães MB, Silva PR, Terrei ALM, Guimarães LOC, Saliba NA. Fluorose dentária em escolares da rede pública na cidade de Araçatuba. Rev Inst Ciênc Saúde 1999; 17:89-92.

10. Núcleo de Estudos e Pesquisas de Sistemas de Saúde/Núcleo de Apoio ao Desenvolvimento de Sistemas de Saúde. Levantamento Epidemiológico em Saúde Bucal: Estado de São Paulo, 1998. São Paulo: Secretaria do Estado da Saúde de São Paulo/Faculdade de Saúde Pública, Universidade de São Paulo; 1999.

11. Silva ALCC, Paiva SM. Ocorrência de fluorose dentária em escolares de Belo Horizonte. Revista do Conselho Regional de Odontologia de Minas Gerais 1995; 1:49-53.

12. Mendonça LL, Kirchner UL, Costa RN, Giovannini JFBG, Pinto MR, Luz MAR. Estudo multicêntrico da fluorose dental e da cárie dental em escolares de 7 e10 anos de Belo Horizonte-Parte I. Revista da Pós Graduação - Universidade Federal de Minas Gerais 1998; 5:101-9.

13. Alcântara CM. Prevalência de fluorose dental em escolares de Curitiba. Revista da Associação Brasileira de Odontologia 1998; 6:304-7.

14. Reis SRA, Mendonça LL, Cabral MBBS, Silva CAL, Souza FM, Fraga IC. Fluorose dental, estado nutricional e cárie dental em escolares de diferentes estratos econômicos de Salvador, Bahia: parte I. Revista da Pós Graduação - Universidade Federal de Minas Gerais 1999; 6:387-94.

15. Secretaria da Saúde do Estado da Bahia. Plano estadual de saúde 1996-2000. Salvador: Secretaria da Saúde do Estado da Bahia; 1996.

16. Fundação Instituto Brasileiro de Geografia e Estatística. Cadastro de cidades e vilas do Brasil, 2000 http://www.ibge.gov.br (acessado em 12/Dez/2000).

17. Secretaria Municipal de Saúde-Salvador. Plano Municipal de Saúde 1994-1997. Salvador: Secretaria Municipal de Saúde; 1994.

18. Ministério da Educação, Instituto Nacional de Estudos e Pesquisas Educacionais. Censo educacional 2000. http://www2.inep.gov.br (acessado em 4/Jan/2001)

19. Cangussu MCT, Castellanos RA, Pinheiro MF, Albuquerque SR, Pinho C. Cárie dentária em escolares de 12 e 15 anos de escolas públicas e privadas de Salvador, Bahia, Brasil, em 2001. Pesqui Odontol Bras 2002; 16:379-84.

20. World Health Organization. Oral health surveys. Basic methods. Geneva: World Health Organization; 1997.

21. Conselho Nacional de Saúde. Resolução 196/96 do Conselho Nacional de Saúde sobre a regulamentação da pesquisa em seres humanos. Brasília: Ministério da Saúde; 1997.

22. Kleinbaum DG, Kupper LL, Morgenstern H. Epidemiologic research. New York: John Wiley \& Sons; 1982.

Recebido em 31/Out/2002

Versão final reapresentada em $4 /$ Jun/2003

Aprovado em 25/Set/2003 